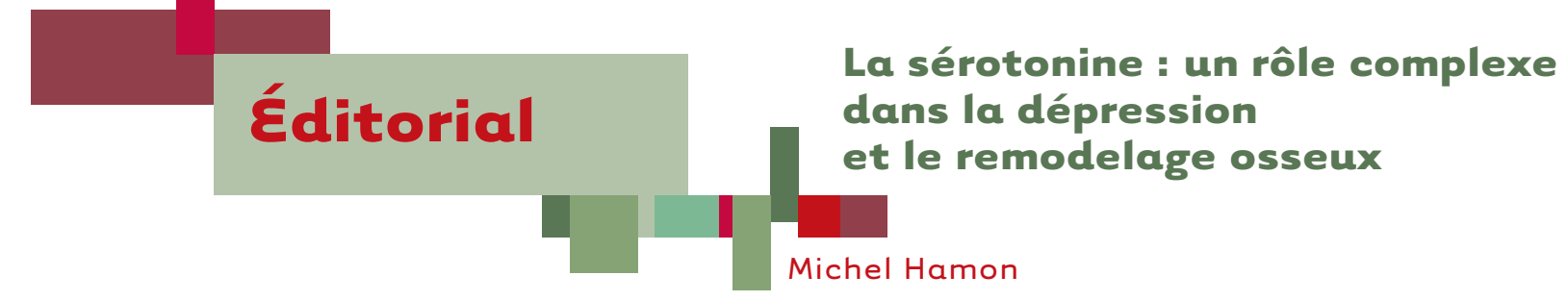

\title{
La sérotonine : un rôle complexe dans la dépression et le remodelage osseux
}

> Parmi les neuromédiateurs, la sérotonine (de dénomination chimique 5-hydroxy-tryptamine ou 5-HT) est certainement I'un des mieux connus, grâce aux - ou à cause des - nombreux articles et reportages que les médias consacrent à la dépression. De fait, il s'agit d'une psychopathologie en constante progression dans nos sociétés libérales. L'Organisation mondiale de la santé (OMS) nous prédit même qu'elle sera, dans 10 ans, la première cause de morbidité chez la femme, la deuxième chez l'homme (après les maladies cardiovasculaires). Et tout le monde sait que les antidépresseurs les plus utilisés aujourd'hui (escitalopram, sertraline, fluoxétine, paroxétine, etc.) sont des inhibiteurs sélectifs de la recapture de la sérotonine (ISRS) dans divers types cellulaires (neurones sérotoninergiques, plaquettes sanguines, lymphocytes, cellules pancréatiques $\beta$, cellules endothéliales, etc.), et que leur action thérapeutique est étroitement liée à leur capacité à élever les taux extracellulaires de cette monoamine dans le cerveau. Cependant, en conclure que la dépression est, au contraire, associée à un déficit de la production de 5-HT dans le système nerveux central (SNC) reste une assertion largement infondée, pour plusieurs raisons. D'abord, parce qu'il s'agit d'une pathologie extrêmement complexe qui fait intervenir de très nombreux facteurs - génétiques, épigénétiques, hormonaux, etc. - et se traduit par des altérations anatomo-fonctionnelles dans plusieurs régions cérébrales comme l'hippocampe, l'amygdale, le cortex préfrontal [1]. Ensuite, parce que la 5-HT n'agit pas d'un seul bloc, par une action positive ou négative, mais exerce des effets multiples via de très nombreux récepteurs codés par une quinzaine de gènes distincts [2]. Ainsi, l'activation de certains d'entre eux, par exemple les $G$ protein coupled receptors (GPCR) de type $5-\mathrm{HT}_{1 \mathrm{~A}}$ et $5-\mathrm{HT}_{1 \mathrm{~B}}$, entraîne une hyperpolarisation membranaire, inhibitrice, alors que celle des types $5-\mathrm{HT}_{2}, 5-\mathrm{HT}_{4}, 5-\mathrm{HT}_{6}$ et $5-\mathrm{HT}_{7}$ déclenche au contraire une dépolarisation, excitatrice. S'agissant des grandes fonctions contrôlées par la 5 -HT, cette dualité d'action va de pair avec l'implication de multiples récepteurs à l'origine d'effets opposés. En l'occurrence, pour ce qui concerne l'action antidépressive des ISRS, l'activation des récepteurs $5-\mathrm{HT}_{1 \mathrm{~A}}$ qui résulte de l'élévation des taux extracellulaires de la 5 -HT est certainement une composante positive importante. $\varepsilon$ n revanche, l'activation des récepteurs $5-\mathrm{HT}_{2 \mathrm{~A}}, 5-\mathrm{HT}_{2 \mathrm{C}}, 5-\mathrm{HT}_{4}$ et $5-\mathrm{HT}_{7}$ est au contraire une composante négative, et, de fait, ce sont les antagonistes de ces récepteurs qui possèdent des potentialités antidépressives. D'où l'idée d'associer dans une même molécule la capacité de bloquer tel ou tel de ces récepteurs à celle d'inhiber la recapture de la 5-HT en vue de développer de nouveaux antidépresseurs plus efficaces.

L'autre raison, majeure (en dehors des aspects économiques, tellement évidents), qui incite à rechercher de telles molécules est la survenue d'effets secondaires souvent gênants et mal supportés (nausées et vomissements, désordes gastroentériques, diminution des capacités sexuelles, etc.) lors d'un traitement par ISRS [3]. Ceux-ci révèlent en fait que la 5-HT est non seulement un neuromédiateur dans le SNC (et pour une population limitée de neurones sérotoninergiques dans le plexus myentérique) mais aussi une neuro-hormone dans de nombreux tissus périphériques où elle y rencontre la plupart de ses récepteurs. La 5 -HT est produite en abondance dans les cellules entérochromaffines de l'intestin, au point qu'on estime que sa quantité dans le SNC ne représente, au mieux, que 3 à $5 \%$ de la quantité totale de 5 -HT chez l'homme comme chez le rat. Comme la barrière hémato-encéphalique empêche tout transport de la 5-HT de la périphérie au SNC et du SNC à la périphérie, il existe donc en réalité deux compartiments distincts, quantitativement très déséquilibrés, au sein desquels la 5-HT exerce des effets propres, voire opposés. Certes, d'autres neuromédiateurs monoaminergiques, comme la dopamine et la noradrénaline, existent aussi à la fois dans le SNC et à la périphérie, mais leur production implique la même enzyme limitante spécifique, en l'occurrence la tyrosine hydroxylase, codée par un seul et même gène dans les neurones et dans les autres types cellulaires (glande médullosurrénale). Dans le cas de la 5 -HT, il existe deux tryptophane hydroxylases, codées par deux gènes distincts: I'une, appelée TPHl, est responsable de la synthèse de 5 -HT dans les tissus périphériques, l'autre, appelée TPH2, est l'enzyme-clé de la synthèse de 5 -HT dans les neurones sérotoninergiques. La délétion spécifique de ces gènes a été réalisée, confirmant le rôle-clé de la 5 -HT cérébrale dans le contrôle des comportements (les souris $\mathrm{TPH}^{-/-}$sont, entre autres, très agressives) et celui de la 5-HT périphérique notamment dans I'homéostasie des fonctions cardiovasculaires et gastroentériques. Ces souris sont particulièrement utiles pour préciser les implications respectives de «ces deux $5-\mathrm{HT}$ », en particulier lorsque les 
données pharmacologiques concluent à des effets opposés, comme par exemple vis-à-vis de la perception de la douleur. À la périphérie, la 5 -HT est clairement pro-nociceptive via l'activation de récepteurs $5-\mathrm{HT}_{2 \mathrm{~A}}$ et $5-\mathrm{HT}_{3}$ présents sur les nocicepteurs, tandis que dans le SNC, en particulier au niveau spinal, elle exerce une action antinociceptive via l'activation de récepteurs $5-\mathrm{HT}_{1 \mathrm{~A}}, 5-\mathrm{HT}_{1 \mathrm{~B}}$ et $5-\mathrm{HT}_{7}$ [4].

Un autre exemple d'action opposée de la 5-HT cérébrale versus périphérique est rapporté par Franck Oury et Gérard Karsenty [5] dans ce numéro de Médecine/Sciences $(\rightarrow)$.

$(\rightarrow)$ Page 713 de ce numéro

Il concerne le remodelage osseux qui assure le renouvellement constant (et complet, en une dizaine d'années chez l'homme) du squelette, sur lequel la 5 -HT cérébrale exerce une influence positive et la 5-HT périphérique une influence négative. Une décalcification et une diminution de la densité et de la masse osseuses ont été rapportées en cas de dépression sévère récurrente, en relation avec l'hypersécrétion de cortisol, de noradrénaline et de cytokines proinflammatoires, voire la baisse du tonus sérotoninergique cérébral, qui caractérisent l'état de stress associé à cette maladie [6]. Pour ce dysfonctionnement aussi, les antidépresseurs ISRS exercent des effets secondaires délétères puisqu'ils contribuent eux-mêmes à accroître la résorption du tissu osseux, en particulier au niveau du col du fémur. De fait, plusieurs études épidémiologiques convergentes montrent une augmentation significative des fractures lors de traitements avec ces médicaments [7]. Ces observations ont bien sûr suscité de nombreux travaux, et l'article de Oury et Karsenty [5] en fait un excellent résumé. Ainsi, il est aujourd'hui clairement établi que les trois types de cellules du tissu osseux, les ostéoblastes (qui fabriquent le tissu osseux), les ostéoclastes (qui le détruisent) et les ostéocytes (issus de la différenciation d'ostéoblastes) possèdent la capacité de synthétiser et de capter la 5-HT et expriment plusieurs types de ses récepteurs [8]. Selon Oury et Karsenty [5], c'est via la stimulation de récepteurs $5-\mathrm{HT}_{1 \mathrm{~B}}$ que la $5-\mathrm{HT}$, présente en excès sous l'action des ISRS, exercerait une action locale inhibitrice sur la prolifération des ostéoblastes, et donc contribuerait à la diminution de la densité osseuse chez les sujets traités par ces antidépresseurs. Dès lors, le blocage de cette action périphérique de la 5 -HT pourrait s'opposer à l'effet négatif des ISRS sur le remodelage osseux. Plusieurs voies sont certainement possibles (en particulier, un antagoniste des récepteurs $5-\mathrm{HT}_{1 \mathrm{~B}}$ qui ne franchirait pas la barrière hémato-encéphalique). Pour leur part, Oury et Karsenty [5] préconisent un blocage des enzymes tryptophane hydroxylases par une molécule qui justement ne franchit pas cette barrière et donc inhibe spécifiquement la TPHI. La synthèse cérébrale de la 5-HT n'étant pas affectée, l'efficacité antidépressive des ISRS est a priori maintenue. Curieusement cependant, les fonctions périphériques qui dépendent étroitement de la 5-HT, notamment la motricité intestinale, ne semblent pas non plus altérées par cet inhibiteur de la TPHl. En réalité, même à des doses élevées, ce composé (LP53401) ne réduit au mieux la synthèse périphérique de 5 -HT que de $50 \%$, ce qui explique sa bonne tolérabilité [9]. Au-delà de la prévention de l'effet délétère des ISRS sur le remodelage osseux, voire de l'ostéoporose post-ménopause [10], qui est mise en avant par Oury et Karsenty [5], on peut espérer que les autres effets secondaires de ces antidépresseurs - évoqués ci-dessus -, voire des anorexigènes sérotoninergiques (hypertension artérielle pulmonaire, valvulopathie cardiaque), qui dépendent entièrement de la 5-HT périphérique, puissent être significativement réduits par le blocage spécifique partiel de la TPHI.

À suivre... $\diamond$

Serotonin: a player in both depression and bone remodeling

\section{CONFLIT D'INTÉRÊTS}

L'auteur déclare n'avoir aucun conflit d'intérêts concernant les données publiées dans cet article.

\section{RÉFÉRENCES}

1. Krugers HJ, Lucassen PJ, Karst H, Joëls M. Chronic stress effects on hippocampal structure and synaptic function : relevance for depression and normalization by anti-glucocorticoid treatment. Front Synaptic Neurosci $2010 ; 2$ : 1-10.

2. Green AR. Neuropharmacology of 5-hydroxytryptamine. BrJ Pharmacol 2006 ; $147:$ S145-52

3. Hamon M, Bourgoin S. Pharmacological profile of antidepressants: a likely basis for their efficacy and side effects? Eur Neuropsychopharmacol 2006 ; 16: S625-32.

4. Kayser V, Bourgoin S, Viguier F, et al. Toward deciphering the respective roles of multiple $5-\mathrm{HT}$ receptors in the complex serotonin-mediated control of pain. In: Beaulieu P, Lussier D, Porreca F, Dickenson AH, eds. Pharmacology of pain. Seattle: IASP Press, $2010:$ 185-206.

5. Oury F, Karsenty $G$. La double vie de la sérotonine ou quand la génétique découvre la physiologie. Med Sci (Paris) $2010 ; 26: 713-8$.

6. Bab I, Yirmiya R. Depression, selective serotonin re-uptake inhibitors and the regulation of bone mass. IBMS BoneKEy $2009 ; 6: 8-15$.

7. Richards JB, Papaioannou A, Adachi JD, et al. Effect of selective serotonin reuptake inhibitors on the risk of fracture. Arch Intern Med 2007 ; 167 : 188-94.

8. Battaglino R, Fu J, Späte U, et al. Serotonin regulates osteoclast differentiation through its transporter. J Bone Miner Res 2004 ; 19 : 1420-31.

9. Liu $Q$, Yang $Q$, Sun W, et al. Discovery and characterization of novel tryptophan hydroxylase inhibitors that selectively inhibit serotonin synthesis in the gastrointestinal tract. J Pharmacol Exp Ther 2008 ; 325 : 47-55.

10. Yadav VK, Balaji S, Suresh PS, et al. Pharmacological inhibition of gut-derived serotonin synthesis is a potential bone anabolic treatment for osteoporosis. Nat Med $2010 ; 16: 308-12$.

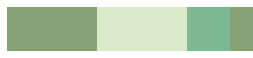

M. Hamon

Inserm U894

Centre de psychiatrie et neurosciences Universités Pierre et Marie Curie et Paris Descartes

Site Pitié-Salpêtrière

91, boulevard de l'Hôpital 75634 Paris Cedex 13, France michel.hamon@upmc.fr

\section{TIRÉS À PART}

M. Hamon 